\title{
Experiência de pacientes com deficiências na utilização de serviços públicos odontológicos
}

\section{Experience of disabled patients in using dental public services}

\author{
Juliana Rodrigues Paulo ${ }^{1}$ \\ Érick TÁssio Barbosa NeVEs ${ }^{2}$ \\ Matheus de França Perazzo ${ }^{3}$ \\ Eliane Medeiros de Batista Serpa ${ }^{4}$ \\ Ana FLÁVIA GranVILLE-Garcia ${ }^{5}$
}

\begin{abstract}
RESUMO
Objetivo: Descrever a experiência de indivíduoscom deficiências matriculados em umaAssociação de Pais e Amigos dos Excepcionais na utilização de serviços públicos odontológicos.

Metodologia: Foi realizado um estudo transversal e analítico com amostra de 121 participantes.Os responsáveis pelos alunosresponderam a um formulário com informaçõessocioeconômicas, clínicas e sobre dificuldades para a utilização dos serviços odontológicos. Foi utilizado o teste Qui-quadrado de Pearsonpara a análise dos dados assumindo como significativos valores de $p<0,05$.

Resultados: Os resultados mostraram uma maior ocorrência deindivíduos com deficiência mental 54 (44,6\%), cuidados pela mãe100 (75,2\%), e já atendidosem serviços públicos odontológicosdo município $62(51,2 \%)$. Para $57(47,1 \%)$ dos participantes,atribuiu-se alguma dificuldadena utilização de serviços públicos odontológicos.Apenas o recebimento de informações sobre locais de atendimento foi associado à utilização dos serviços $(p<0,05)$.

Conclusão: A ocorrência de indivíduos com dificuldades na utilização de serviços públicos odontológicos foi alta e os responsáveis informados sobre locais para o atendimento frequentaram mais esses serviços.

Palavras-chave: Assistência Odontológica para Pessoas com Deficiências.Serviços de Saúde Bucal. Utilização.
\end{abstract}

\begin{abstract}
Objective: The aim of this study was to describe the experience of patients with special needs enrolled in an Association of Parents and Friends of Exceptional Children in the utilization of public dental services.

Methodology: It was a cross-sectional analytical study with a sample of 121 participants. Caregivers answered a form with socioeconomic and clinical information and difficulties about utilization of dental services. Chi-square test of Pearson was used for data analysis assuming as significant, values of $p<0.05$. Results: For this study, there was a higher prevalence of individuals with mental disabilities 54 (44.6\%), cared by mothers $100(75.2 \%)$ and those who haveused public dental services in the city $62(51.2 \%) .57$ (47.1\%) participants had some difficulty in using public dental services. Receiving information about service sites was the only variable associated with usage of dental public services $(p<0.05)$.

Conclusion: It can be concluded that the occurrence of individuals with difficulties in using public dental services was high and people informed about places to receive dental careattended morefrequently to these services.
\end{abstract}

Key-words: Dental Care for Disabled.Dental Health Services. Utilization.

Cirurgiã-dentista graduada pela Universidade Federal da Paraíba (UFPB), email: julipaulo@hotmail.com,

Mestrando em Odontologia pela Universidade Estadual da Paraíba (UEPB), email: erick.tassio@hotmail.com,

Doutorando em Odontologia pela Universidade Federal de Minas Gerais (UFMG), email:matheusperazzo@hotmail.com,

Professora da Universidade Federal da Paraíba (UFPB) e doutora em Odontopediatria pela Universidade de Pernambuco (UPE), email: elibmedeiros@yahoo.com.br,

5 Professora da Universidade Estadual da Paraíba (UEPB) e doutora em Odontopediatria pela Universidade de Pernambuco (UPE), email:anaflaviagg@hotmail.com, contato: (83) 33153326. 


\section{INTRODUÇÃO}

A Organização Mundial de Saúde (OMS) estima que um a cada dez indivíduos apresenta algum tipo de deficiência. ${ }^{1}$ Relatos nacionais revelam que cerca de $23,9 \%$ da população brasileira apresentam algum tipo de deficiência visual, auditiva, motora, mental ou intelectual. Entretanto,apesar da presença de recursos de saúde disponíveis para atendê-los, o acesso é dificultado por preconceitos, discriminação, negligência e pela falta de conhecimento sobre as dificuldades enfrentadas por essa população. ${ }^{2}$

Pacientes Portadores de Necessidades Especiais (PNE's) demandam maiores cuidados devido às suas limitações físicas, mentais, sociais, sensoriais, neurológicas e/ ou emocionais, e mais de dois terços dessa população não recebem qualquertipo de cuidado de saúde bucal. ${ }^{2-4}$

Problemas de saúde bucal são comuns em indivíduos com necessidades especiais e apresentam alta prevalência. Situações clínicas como as doenças periodontais $(53,1 \%$ a $80,3 \%),{ }^{5-6}$ a má-oclusão $(93 \%)^{7}$ e a cárie dentária $(32,2 \% \text { a } 80 \%)^{6,8}$ são frequentes, principalmente em indivíduos com limitações neuropsicomotoras. Em consequência, há uma grande necessidade de tratamentos preventivos e reabilitadores, na maioria das vezes dificultados devido à complexidade no manejo do paciente..$^{2,4}$

As práticas curativas empregadas na Odontologia parecem muitas vezes exceder as medidas preventivas e sugerem que há a descontinuidade da atenção odontológica para os pacientes com necessidades especiais. $O$ atendimento, portanto, é predominantemente emergencial e a cárie e a dor dentária destacam-se como sendoas principais razões para a busca pelos serviços de saúde..$^{9-11}$

Embora disparidades no recebimento de cuidados odontológicos sejam mais frequentes entre pessoas com necessidades especiais, sabe-se pouco sobre a utilizaçãodos serviços odontológicos por esses pacientes e estão relativamente escassos dados que retratem a situação de diferentes localidades. ${ }^{11-13}$

Diante do exposto, o presente estudo teve como objetivodescrevera experiência de pacientes com necessidades especiais matriculados em uma Associação de Pais e Amigos dos Excepcionais na utilização de serviços públicos odontológicos.

\section{MATERIAIS E MÉtodos}

Foi realizado um estudo observacional do tipo transversal e analítico envolvendo uma amostra de 121 responsáveis pelos indivíduos com necessidades especiais entre 1 e 59 anos de idade devidamente matriculados na Associação de Pais e Amigos dos Excepcionais de João Pessoa (APAE/PB) e frequentando regularmente a mesma.

O presente estudo foi conduzido de acordo com a declaração de Helsinque e com base na resolução 196/96 do Ministério da Saúde, sendo independentemente revisado e aprovado pelo Comitê de Ética em Pesquisas com Seres Humanos do Hospital Universitário Lauro Wanderley, aprovado sob o protocolo $\mathrm{n}^{\circ}$. 104/09. Além disso, foi solicitada a autorização da presidente da Comissão de Pesquisa da Associação dos Pais e Amigos dos Excepcionais (APAE/PB). Todos os direitos dos participantes foram garantidos. Os participantes receberam informações com relação aos objetivos do estudo e assinaram um Termo de Consentimento Livre e Esclarecido previamente à participação no estudo.

A Associação de Pais e Amigos dos Excepcionais de João Pessoa (APAE/PB) é uma entidade filantrópica, de caráter cultural, assistencial e educacional, sem fins lucrativos, que promove a melhoria da qualidade de vida das pessoas com deficiência, em seus ciclos de vida desde quando crianças, adolescentes e adultos até idosos, buscando assegurar-lhes o pleno exercício da cidadania. ${ }^{14}$

Essa instituição foi selecionada pelo fato de ser referência para o Estado paraibano 
no atendimento de pacientes portadores de necessidades especiais de todo município e ainda assim não possuir um setor de atenção odontológica próprio, necessitando recorrer frequentemente a encaminhamentos para os serviços públicos de saúde da região.

Foram incluídos neste estudo indivíduos com diagnóstico médico prévio dealterações do padrão de normalidade de sua condição física, mental, orgânica e/ou de socializaçãoedevidamente matriculados na instituição APAE.

Um estudo piloto foi conduzido para avaliar a metodologia e compreensão do formulário com $12(10 \%)$ dos responsáveis pelos PNE's da APAE. Diante da ausência de dúvidas sobre os formulários ou acerca da metodologia aplicada no estudo, não foram necessárias alterações no processo de coleta dos dados. Os indivíduos do estudo piloto, foram incluídos na amostra.

A coleta dos dados foi realizada por um único pesquisador previamente treinado para a aplicação do formulário e ocorreu entre setembro e dezembro de 2009. Utilizou-se para essa finalidade um formulário baseado no instrumento apresentado por Pádua (2004), ${ }^{15}$ que envolve em sua composição questões objetivas e subjetivas acerca do tema em estudo. O formulário constou de duas partes: a primeira, com questões direcionadas aos dados sociodemográficos dos indivíduos, no qual foram incluídas a idade, o gênero, tipo de deficiência apresentada, o local e o tempo de tratamento. A segunda parte envolveu aspectos relativos à utilização e participação dos pacientes na atenção odontológica pública. A classificação empregada para os tipos de deficiência foi a mesma desenvolvida por Mugayar (2000). ${ }^{16}$

A amostra foi inicialmente caracterizada por meio de estatística descritiva. A análise dos dados foi realizada utilizando o programa Statistical Package for the Social Sciences (IBM SPSS para Windows, na versão 18.0). A associação entre as variáveis foi avaliada pelo teste Qui-quadrado de Pearson, considerando um nível de significância estatística de 5\%.

\section{Resultados}

Umtotal de 121 participantes permaneceram nesse estudo após a aplicação dos critérios de elegibilidade, sendo que destes, 60 (49,6\%) eram do sexo masculino e $61(50,4 \%)$ do sexo feminino. A idade dos participantes variou entre 1 e 59 anos e as faixas etárias predominantes foram as de 5 a 9 anos $(28,1 \%)$ e acima de 20 anos $(30,6 \%)$.

Em relação ao tempo de permanência na $A P A E$, verificou-se que a maioria dos indivíduos $(82,6 \%)$ estava na instituição havia mais de 13 meses.

As deficiências de maior prevalência na amostra foram a deficiência mental $(44,6 \%)$, seguida da paralisia cerebral $(23,1 \%)$ e da Síndrome de Down (20,6\%), (Tabela 1).

Tabela 1 - Caracterização dos pacientes quanto ao diagnóstico da deficiência.

\begin{tabular}{l|r|c}
\hline DIAGNÓSTICO & $\mathrm{n}$ & $\%$ \\
\hline DESVIO DE INTELIGÊNCIA & & \\
\hline Deficiência Mental & 54 & 44,6 \\
\hline DEFICIÊNCIA FÍSICA & & \\
\hline Paralisia cerebral & 28 & 23,1 \\
\hline Outros defeitos motores & 02 & 1,7 \\
\hline DEFEITO CONGÊNITO/GENÉTICO & & \\
\hline Síndrome de Down & 25 & 20,6 \\
\hline Síndrome de Apert & 01 & 0,8 \\
\hline Síndrome de Rett & 01 & 0,8 \\
\hline Síndrome de Seckel & 01 & 0,8 \\
\hline Microcefalia Congênita & 01 & 0,8 \\
\hline DESVIO COMPORTAMENTAL & & \\
\hline Autismo & 02 & 1,7 \\
\hline DEFICIÊNCIA DE & & \\
AUDIOCOMUNICAÇÃO & & \\
\hline Distúrbio na comunicação oral (fala) & 02 & 1,7 \\
\hline Distúrbio de audiocomunicação & 02 & 1,7 \\
(surdo) & & \\
\hline NÃO DIAGNOSTICADA & 02 & 1,7 \\
\hline TOTAL & & 100,0 \\
\hline
\end{tabular}


Os resultados demonstraram que,em muitos dos casos, a mãe era a principal cuidadora $(75,2 \%)$, sendo o segundo cuidador mais frequente o pai $(10,7 \%)$. Amaioria dos cuidadores apresentou o ensino fundamental incompleto como grau de instrução $(19,8 \%)$ e uma renda familiar de até um salário mínimo (60,3\%).

Uma parcela significativa dos pacientes já havia sido atendida pelos serviços públicos odontológicos do município de João Pessoa $(51,2 \%)$ e a maior parte destes $(69,4 \%)$ frequentou o serviço no nível da Atenção Básica, ocorrendo a última utilização do serviço no decorrer do ano de 2009 (61,3\%).

Uma considerável quantidade de responsáveis $(47,1 \%)$ atribuiu alguma dificuldade em utilizar um serviço odontológico público. Destacaram-se entre as principais queixas e limitações a falta de disponibilidade de vagas para o atendimento odontológico (45,6\%), a falta de vontade/colaboração por parte do próprio paciente $(21,0 \%)$ e a de o cirurgião-dentista negar atender ao paciente com necessidades especiais (17,5\%), (Tabela 2$)$.

Aproximadamente metade dos cuidadores entrevistados 60 (49,6\%) recebeu informações sobre locais específicos para o atendimento odontológico dos pacientes com deficiências. Dos responsáveis informados, 16 (26,7\%) relataram essa indicação por meio de amigos, seguidos daqueles que receberam informação através dos familiares e do cirurgião dentista da Estratégia de Saúde da Família (ESF), ambas as categorias com ocorrência equivalente a $10(16,7 \%)$, cada uma. Alguns pacientes receberam essas informações por meio dos agentes comunitários de saúde responsáveis pela área domiciliada, totalizando $9(15 \%)$ e uma parcela equivalente recebeu informações pelo diretor da APAE, perfazendo o mesmo percentual. Percebeu-se, entretanto, uma participação mais discreta do médico da Estratégia de Saúde da Família nesse processo, que foi de 6 (10\%).
Tabela 2- Distribuição dos PNE's de acordo com a dificuldade de utilização dos serviços públicos odontológicos.

\begin{tabular}{|c|c|c|}
\hline $\begin{array}{l}\text { DIFICULDADES NA UTILIZAÇÃO } \\
\text { DE SERVIÇOS PÚBLICOS } \\
\text { ODONTOLÓGICOS }\end{array}$ & $\mathrm{n}$ & $\%$ \\
\hline $\begin{array}{l}\text { Encontrou dificuldade para } \\
\text { ser atendido nas consultas } \\
\text { odontológicas? }\end{array}$ & & \\
\hline Sim & 57 & 47,1 \\
\hline Não & 47 & 38,9 \\
\hline Nunca procurou & 17 & 14,0 \\
\hline $\begin{array}{l}\text { Qual o motivo da dificuldade } \\
\text { apresentada? }\end{array}$ & & \\
\hline $\begin{array}{l}\text { Falta de vagas para o atendimento } \\
\text { odontológico. }\end{array}$ & 26 & 45,6 \\
\hline $\begin{array}{l}\text { Falta de colaboração por parte do } \\
\text { paciente. }\end{array}$ & 12 & 21,0 \\
\hline $\begin{array}{l}\text { Profissional negou o atendimento } \\
\text { odontológico. }\end{array}$ & 10 & 17,5 \\
\hline $\begin{array}{l}\text { Não possuir serviço odontológico } \\
\text { próximo à residência. }\end{array}$ & 02 & 3,5 \\
\hline $\begin{array}{l}\text { Não conhecer locais para } \\
\text { atendimento odontológico para } \\
\text { pacientes especiais. }\end{array}$ & 02 & 3,5 \\
\hline $\begin{array}{l}\text { Custo alto para o transporte até o } \\
\text { local de atendimento. }\end{array}$ & 02 & 3,5 \\
\hline $\begin{array}{l}\text { Não foi esclarecido sobre a } \\
\text { necessidade de fazer consulta/ } \\
\text { prevenção } \\
\text { em saúde bucal no paciente. }\end{array}$ & 01 & 1,8 \\
\hline $\begin{array}{l}\text { Não possuir tempo disponível para } \\
\text { acompanhar o paciente. }\end{array}$ & 01 & 1,8 \\
\hline Outros motivos. & 01 & 1,8 \\
\hline TOTAL & 57 & 100,0 \\
\hline
\end{tabular}

\section{Verificou-se uma associação} estatisticamente significativa entre as informações de locais e a utilização dos serviços públicos odontológicos $(p<0,05)$, pelo teste estatístico do Qui-quadrado de Pearson, podendo-se dizer que os responsáveis que tiveram informação procuraram mais o serviço público odontológico (Tabela 3). 
Tabela 3 - Associação das informações sobre locais de atendimento odontológico para pacientes com necessidades especiais e a utilização de serviços públicos odontológicos.

\begin{tabular}{c|c|c|c}
\hline & \multicolumn{2}{|c|}{ RECEBIMENTO DE INFORMAÇÕES } & p-VALOR \\
\hline UTILIZAÇÃO DOS SERVIÇOS & $\operatorname{Sim}$ & Não & \multirow{2}{*}{$\mathrm{p}=0,001$} \\
\hline Sim & 36 & 26 & \\
\hline Não & 24 & 35 & \\
\hline
\end{tabular}

${ }^{*}$ p-valor obtido por meio do teste Qui-quadrado de Pearson

Não foi encontrada uma associação estatisticamente significativa entre o tipo de deficiência e a utilização de serviços públicos odontológicos, como para as informações sobre locais de atendimento odontológico e o grau de escolaridade dos responsáveis pelos PNE's da APAE $(p>0,05)$.

\section{Dıscussão}

A deficiência mental foi a deficiência de maior ocorrência para este estudo (44,6\%). Outras investigações realizadas em ambientes institucionalizados observaram resultados semelhantes, destacando-se com uma maior ocorrência os distúrbios neurológicos $(25,1 \%$ a $60,3 \%)$ e as deficiências intelectuais $(64 \%) .8,9,11,17$

Com relação aos serviços públicos odontológicos de saúde, observou-se que não foram utilizados por $48,8 \%$ dos PNE's. Em concordância com esse achado, Santos et al. $(2008)^{18}$ estimaram que $42,8 \%$ dos pacientes com necessidades especiais não usaram o serviço público odontológico ofertado. Um número menor foi verificado em um estudo conduzido por Flório et al. $(2007)^{19}(37,5 \%)$. Essa variação pode ser resultado do critério empregado pelo autor para a classificação dos indivíduos que não utilizaram os serviços odontológicos. Para o referido trabalho, o autor considerou de forma geral como "não utilização" dos serviços odontológicos os indivíduos que nunca foram ao dentista, independentemente da natureza desse serviço, público ou privado, enquanto o atual trabalho preocupou-se de maneira particular apenas com a utilização dos serviços públicos.

Para este estudo, a maioria $(45,6 \%)$ dos responsáveis relatou dificuldade de utilização dos serviços odontológicos pela falta de disponibilidade de vagas. Uma justificativa para essa possível escassez de vagas disponíveis para o uso de serviços odontológicos pelos PNE's poderia ser a natureza da intervenção, muitas vezes de alta complexidade, o que faz que alguns profissionais da rede básica encontrem problemas no atendimento desse perfil de paciente e acabem negando o atendimento, sobrecarregando os serviços especializados. Alguns cirurgiões-dentistas não atendem por completo às necessidades desse grupo, recorrendo ao encaminhamento dos pacientes para outros serviços. ${ }^{20}$

Sabe-se que o atendimento a pacientes com necessidades especiais gera ansiedade tanto para o dentista como para o paciente, tornandose mais evidente quandoo profissional não apresenta preparo técnico. ${ }^{21}$ Por essa razão, acreditamos que a problemática de recusa ao atendimento está mais fortemente ligada ao nível da atenção básica, uma vez que os serviços especializados, como o CEO (Centro de Especialidades Odontológicas), contam com especialistas na área.

É importante, nessas condições, a ampliação de programas e políticas públicas direcionados para o atendimento dos pacientes com necessidades especiais, ${ }^{8,11,17}$ ressaltando o papel do Cirurgião-Dentista 
na atenção básica em fornecer suporte aos PNE's, acolhendo-os, prevenindo e tratando os agravos presentes, toda vez que possível. Merece destaque também o fortalecimento na formação profissional dos recursos humanos em Odontologia durante a graduação ou mesmo nos cursos de pós-graduação. ${ }^{22-23}$

Outra limitação exposta pelos paisresponsáveis dos pacientes especiais usuários dos serviços públicos odontológicos, neste estudo, foi a falta de colaboração por parte dos pacientesem receber o tratamento $(21,0 \%)$. Esse dado corrobora os achados de Cumella et al. (2000), ${ }^{24}$ que relatam a existência de atitudes agressivas, medo e falta de colaboração durante procedimentos odontológicos e dificuldades encontradas pelos responsáveis nos cuidados básicos de saúde do paciente. Tais aspectos indicam a necessidade de uma reavaliação dos métodos e técnicas empregados para a motivação, educação e sensibilização do indivíduo e/ou cuidadores. ${ }^{24}$

Em um estudo do tipo caso-controle, Collado et al. (2008) ${ }^{25}$ relataram que PNE'sapresentaram cinco vezes mais chances de desenvolver dificuldades durante o tratamento odontológico quando comparados aos indivíduos do grupo controle. Os autores disseram também que a dificuldade em higienizar os dentes foi de aproximadamente quinze vezes maior para os indivíduos com necessidades especiais em comparação com os controles. Ressalta-se dessa forma o papel fundamental do cuidador na saúde bucal do indivíduo com necessidades especiais. Nesse sentido, Risma et al. $(2015)^{26}$ sugerem uma relação diretamente proporcionalentre o nível desegurança dos cuidadores para executar atividades que envolvem o manejo dos PNE'se sua formação e preparo para desempenhar tais funções.

Mais da metade dos pacientes deste estudo já foram atendidos pelos serviços públicos odontológicos ofertados na cidade, sendo que desses,69,3\% foram atendidos na atenção básica. Os resultados sugerem que a porta de entrada do usuário ao sistema público de saúde tem sido primordialmente a atenção primária e devido à considerável demanda há maiores chances de barreiras geográficas e operacionais. ${ }^{27}$

Em um estudo realizado em Minas Gerais, estabeleceu-se o perfil dos pacientes atendidos sob sedação ou anestesia geral como predominantemente de adultos, do sexo masculino e com diagnóstico de transtornos mentais ou comportamentais. ${ }^{9}$ Tais resultados podem sugerir que certos pacientes especiais com condições mais incapacitantes utilizariam menos o atendimento público odontológico no nível da atenção primária, recorrendo à atenção especializada ou terciária como alternativa, sendo a anestesia geral relatada como técnica mais confortável, como percebese em alguns estudos. ${ }^{11,28}$ Para este estudo, o tipo de deficiência não foi associado ao atendimento nos serviços públicos odontológicos, provavelmente pelo tamanho da amostra e a ausência do exame clínico para determinar as necessidades de cada paciente. A aplicação de exames não foi conduzida, pois não contemplava o objetivo deste trabalho.

Os CEO's (Centros de Especialidades Odontológicas) foram implantados pela portaria $n^{\circ}$. 599/GM de 23 de março de 2006,com a finalidade de atender a especificidades da população no atendimento odontológico quando encaminhados por meio da atenção básica. ${ }^{29}$

Neste estudo, o recebimento de informações sobre locais de serviços foi associadoà utilização de serviços públicoso dontológicos,indicando que os usuários cujos responsáveis tiveram acesso à informação utilizaram mais o serviço.

Um estudo realizado por Ohtawaet al. $(2012)^{30}$ estimou que $62,6 \%$ dos indivíduos 
com necessidades especiais atendidos em ambiente hospitalar foram previamente encaminhados através de outros serviços de saúde. Tal achado indica a importância da atividade de referência e contrarreferência situada no nível de atenção básica pública para oferta dos serviços odontológicos, atividade consolidada, portanto, pelo recebimento de informações.

Uma possível limitação deste estudo foi a amostra por conveniência. Entretanto, a Associação de Pais e Amigos dos Excepcionais de João Pessoa-Paraíba é a entidade de destaque no atendimento a pacientes especiais para a cidade em questão e é uma referência para o Estado.

Sugerimos ainda a realização de estudos para a avaliação do padrão de distribuição dos tratamentos odontológicos recebidos por pacientes especiais em diferentes serviços, por especialidade, de forma a possibilitar a formulação de políticas direcionadas para a prevenção dos agravos de maior ocorrência para esses indivíduos.

\section{Considerações finaIS}

A ocorrência de indivíduos com necessidades especiais que apresentaram dificuldade na busca de atendimento odontológico foi alta, sendo a atenção básicao nível de atendimentomais procurado.Para este estudo, o recebimento de informações sobre locais de atendimento exerceu impactona utilização dos serviços públicos odontológicos pelos pacientes com necessidades especiais.

\section{REFERÊNCIAS}

1. Secretaria de Direitos Humanos da Presidência da República (SDH/PR)/Secretaria Nacional de Promoção dos Direitos da Pessoa com Deficiência (SNPD)/Coordenação-Geral do Sistema de Informações sobre a Pessoa com Defi- ciência, Cartilha do censo 2010. Pessoas com Deficiência. Brasília-PR, SNPD, 32p., 2012.

2. Organização Mundial de Saúde. Relatório mundial sobre a deficiência. Secretaria dos direitos da pessoa com deficiência.São Paulo, 334p, 2012.

3. WilliansJJ,SpanglerCC,Yusaf NK. Barriers to dental care access for patients with special needs in an affluent metropolitan community. Spec Care Dent. 2015; 35(4): 190-6.

4. Rapalo DM, Davis JL, Burtner P., Bouldin ED. Cost as a barrier to dental care among people with disabilities: a report from the Florida behavioral risk factor surveillance system. Spec Care Dent. 2010;30(4):133-9.

5. Morales-Chávez M.,Rada-BerroteranA.,ArcilaRamos L. Periodontal status of mentally handicapped school children in Caracas, Venezuela. A cross sectional study. Journal of Oral Research.2014;3(3): 156-161.

6. Morgan JP, Minihan PM, Stark $P C$,FinkelmanMD, Yantsides KE, Park A. et al. The oral health status of 4,432 adults with intellectual and developmental disabilities. J Am Dent Assoc. 2012;143(8): 838-846.

7. Vellappally S., Gardens SJ,Al KheraifAAA,Krishna M.,Babu S., Hashem M. et al. The prevalence of malocclusion and its association with dental caries among 12-18-year-old disabled adolescents. BMC Oral Health. 2014; 123(14):1-7.

8. Pereira LM, Mardero E., Ferreira SH, Kramer $\mathrm{PF}$, Cogo RB.Atenção odontológica em pacientes com deficiências: a experiência do curso de Odontologia da ULBRA Canoas/RS. Stomatos, 2010; 31 (8):92-99.

9. Santos JS,Valle DA,Palmier AC,Amaral JHL, Abreu MHNG. Availability of hospital dental care services under sedationor general anesthesia for individuals with special needs in the Unified Health System for the State of Minas Gerais (SUS-MG), Brazil.Cien Saude Colet. 2015; 20(2): 515-524.

10. Badre B.,Serhier Z., Elarabi S. Waiting times before dental care under general anesthesia in children with special needs in the children's Hospital of Casablanca.Pan Afr Med J. 2014;20(17):298. 
11. Castro AM,Marchesoti MGN,Oliveira FS, Novaes MSP. Avaliação do tratamento odontológico de pacientes com necessidades especiais sob anestesia geral. RevOdontol UNESP. 2010; 39(3):137-142.

12. Rocha LL,Saintrain MVL, Vieira-Meyer PGF. Access to dental public services by disabled persons. BMC Oral Health. 2015;15 (35):1-9.

13. Douglas CW,GassmanP. The oral health of vulnerable older adults and persons with disabilities.Spec care in Dentist. 2013; 33(4):156-163.

14. Associação de Pais e Amigos dos Excepcionais(APAE), João Pessoa-PB. Disponível em:<htpp://joaopessoa.apaebrasil.org.br/ artigo.phtml?a=13108>. [acesso em 6 de dezembro de 2015].

15. Pádua EMM. Metodologia da pesquisa: abordagem teórico-prática, 10. ed. Rev. e atual. Campinas, SP: Papirus; 2004.

16. Mugayar LRF. Pacientes portadores de necessidades especiais. In:Manual de odontologia e saúde oral, 1. ed. São Paulo: Pancast; 2000,p. 131-76.

17. Queiroz FS,Rodrigues MMLF,Cordeiro Junior GA,Oliveira AB, Oliveira JD,Almeida ER. Avaliação das condições de saúde bucal de portadores de necessidades especiais. Rev Odontol UNESP. 2014; 43 (6): 396-401.

18. Santos BMO,Diolinda JNA,Fernandes DR. Perfil Epidemiológico dos portadores de necessidades especiais atendidos em uma clínica odontológica.RBPS. 2008; 21(2):83-91.

19. Flório FM,Basting RT,Salvatto MV, Migiato KL. Saúde bucal em indivíduos portadores de múltiplas deficiências.RGO. 2007; 55(3):251-256.

20. Lawrence H.,Sousa LP,Gonçalves FL,Saintrain MVL, Vieira APGF. Acesso à saúde bucal pública pelo paciente especial: a ótica do Cirurgião-Dentista.Rev Bras Promoç Saúde. 2014; 27(2): 190-197.

21. Possobon RF,Carrascoza KC,Moraes ABAE,Costa JAL. O tratamento odontológico como gerador de ansiedade.Psicologia em Estudo. 2007;13(3):609-616.

22. Clemetson JC,Jones DL,Lacy ES, Hale D., Bolin KA. Preparing dental students to treat patients with special needs: changes in predoctoral education after the revised accreditation standard.J Dent Educ. 2012;76(11):1.457-1.465.

23. Ahmad S.,Razak IA, Borromeo GL. Undergraduate Education in Special Needs Dentistry in Malaysian and Australian Dental Schools. J Dent Educ. 2014;78 (8):1.154-1.161.

24. Cumella S.,Ransford N.,Lyons J., Burnham $\mathrm{H}$. Needs for oral care among people with intellectual disability not in contact with community dental services.J Intellect Disabil Res. 2000;44(1):45-52.

25. Collado V.,Faulks D., Hennequin M. A survey of the difficulties encountered during routine hygiene and health care by persons with special needs.Disabil Rehabil. 2008;30(14): 1.047-54.

26. Risma KM, Weber-Gasparoni K.,Swenson SE,Ettinger RL, Qian F. Group home caregivers' comfort levels regarding physical resistance during oral hygiene care.Spec Care Dentist. 2015; 35(3):123-31.

27. Chaves SCL,Soares FF, Rossi TRA,CangussoMCT,Figueredo ACL, Cruz DN, Cury PR. Características do acesso e utilização de serviços odontológicos em municípios de médio porte.Cien Saude Colet. 2012; 17 (11): 3.115-3.124.

28. Molina GF,Faulks D., Frencken J. Acceptability, feasibility and perceived satisfaction of the use of atraumatic restorative treatment approach for people with disability.Braz Oral Res [on-line]. 2015; 29(1): 1-9.

29. Centro de Especialidades Odontológicas (CEO).Disponível em: <dab.saude. gov.br/portaldab/ape_brasil_sorridente. php? conteudo $=$ ceo $>$. [acesso em 6 de dezembro de 2015].

30. Ohtawa Y., Tsujino K.,Kubo S., Ikeda M. Dental treatment for patients with physical or mental disability under general anesthesia at Tokyo Dental College Suidobashi Hospital.Bull Tokyo Dent Coll. 2012; 53(4):181-7.

Submetido em: 18-2-2017

Aceito em: 6-6-2017 